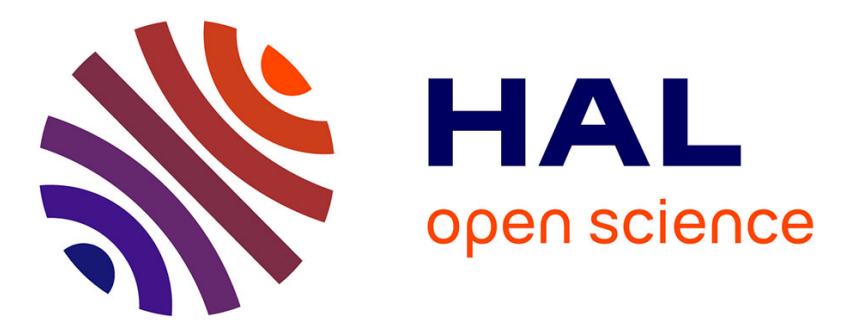

\title{
Optimal Force Generation of 3-RRR Decoupled Planar Robots for Ensuring Unlimited Platform Rotation
}

\author{
Sébastien Briot, Vigen Arakelian, Damien Chablat, Philippe Wenger
}

\section{To cite this version:}

Sébastien Briot, Vigen Arakelian, Damien Chablat, Philippe Wenger. Optimal Force Generation of 3-RRR Decoupled Planar Robots for Ensuring Unlimited Platform Rotation. Romansy 19 - Robot Design, Dynamics and Control, Springer, pp.109-116, 2012, CISM International Centre for Mechanical Sciences, 978-3-7091-1378-3. 10.1007/978-3-7091-1379-0_14 . hal-00687694

\section{HAL Id: hal-00687694 https://hal.science/hal-00687694}

Submitted on 25 Jun 2019

HAL is a multi-disciplinary open access archive for the deposit and dissemination of scientific research documents, whether they are published or not. The documents may come from teaching and research institutions in France or abroad, or from public or private research centers.
L'archive ouverte pluridisciplinaire HAL, est destinée au dépôt et à la diffusion de documents scientifiques de niveau recherche, publiés ou non, émanant des établissements d'enseignement et de recherche français ou étrangers, des laboratoires publics ou privés. 


\title{
Optimal Force Generation of 3-RRR Decoupled Planar Robots for Ensuring Unlimited Platform Rotation
}

\author{
Sébastien Briot ${ }^{\dagger}$, Vigen Arakelian ${ }^{\ddagger}$, Damien Chablat ${ }^{\dagger}$, \\ and Philippe Wenger ${ }^{\dagger}$ \\ † Institut de Recherche en Communications et Cybernétique de Nantes \\ (IRCCyN) UMR CNRS 6592, Nantes, FRANCE \\ ¥ Institut National des Sciences Appliquées de Rennes (INSA) \\ M\&CSE Department, Rennes, FRANCE
}

\begin{abstract}
This paper deals with the optimal dynamics of the 3RRR decoupled robot in which the linear displacements of the platform are decoupled from its orientation. The particularity of the 3 -RRR decoupled robot is that an optimal choice of the leg's geometric parameters allows an unlimited platform rotation, which can be attractive for many industrial applications. However, a singular configuration is necessarily encountered during full platform rotation. In order to generate a stable motion in the presence of singularities, optimal dynamic conditions are disclosed. The suggested optimal conditions ensuring unlimited platform rotation are illustrated and validated by numerical simulations with ADAMS.
\end{abstract}

\section{Introduction}

In the last few decades, parallel manipulators have been the subject of various studies. Many problems devoted to their singularity, kinematics and dynamics properties were disclosed. Parallel manipulators are used in more and more applications nowadays. Consequently, new research problems are continuously appearing. One of the structural disadvantages of parallel manipulators is the low platform rotation capability due to the multi-branch architecture. In the last years this problem has been studied by several researchers. In (Liu et al., 2005) a new family of 3-DOF parallel manipulators with high rotational capability was proposed. The platform orientation range was increased by non symmetric placement of three non-identical legs. In (Sacludean et al., 2002; Company et al., 2005; Nabat et al., 2006b) new architectures of 3-DOF parallel manipulators with unlimited rotation of the end-effectors were developed. They consist of a pair of connected five-bar 
mechanisms. A similar approach was applied in a parallel robot for medical 3D-ultrasound imaging (Lessard et al., 2008, 2007). In the parallel manipulator H4, a complementary gear transmission (Kokikabushiki et al., 2000) together with a belt transmission (Nabat et al., 2006a) were used to amplify the orientation angle of the end-effector. A new family of spatial parallel mechanisms with decoupled and unlimited rotation of the moving platform was proposed in (Gogu, 2009). In the suggested architectures the moving platform performs two independent translations and one independent unlimited rotation whose axis is perpendicular to the plane of translation.

A modified mobile platform design (Fig. 1a) was proposed in a recent study (Arakelian et al., 2011), thus decoupling the position and the orientation of the platform. In the 3-RRR decoupled robot, the loop $\left(O_{1}, A_{1}\right.$, $\left.C, A_{2}, O_{2}\right)$ moves point $C$ and the leg $\left(O_{3}, A_{3}, B_{3}\right)$ adjusts the orientation $\phi$ according to the position. If the position of point $C$ is given, this third leg is equivalent to a four-bar linkage, which ensures a full rotation of the platform. For the generation of a full rotation of the platform, however, the four-bar linkage must cross a singularity.

In this paper, the optimal dynamic conditions are found in order that the 3-RRR decoupled robot can cross a singularity during a full rotation motion of its platform.

\section{Description of the 3-RRR Decoupled Robot}

\subsection{Structural features and singularities}

Let us consider the architecture of the suggested manipulator (Fig. 1a). As shown in Fig. 1a, axis $\mathbf{x}_{\mathbf{0}}$ is along vector $\overrightarrow{O_{1} O_{2}}$. The lengths of link $O_{i} A_{i}$ (resp. $A_{i} B_{i}$ ) are denoted as $L_{1 i}$ (resp. $L_{2 i}$ ). The length of the platform $C B_{3}$ is denoted as $R$. The positions of the base joints $O_{i}$ along $\mathbf{x}_{\mathbf{0}}$ and $\mathbf{y}_{\mathbf{0}}$ are denoted as $\left(x_{O_{i}}, y_{O_{i}}\right)$, with $x_{O_{1}}=y_{O_{1}}=y_{O_{2}}=0$.

The aim of such a modification of the 3-RRR robot is to obtain an unlimited platform rotation. Indeed, the four-bar linkage, which generates the rotation of the platform, is equivalent to a rocker-crank mechanism. In this case, it is able to transfer the oscillation of the input link $O_{3} A_{3}$ to the full rotation of the platform $C B_{3}$. The robot should be designed so that this condition is satisfied in the whole workspace. However, a singularity must be encountered during a full platform rotation motion. This singularity occurs when $C B_{3}$ and $A_{3} B_{3}$ are aligned (Fig. 1b). As a result, the optimal generation of the input link $O_{3} A_{3}$ for passing through singular configurations must be achieved (Briot and Arakelian, 2008). This approach is based on the study of the robot dynamic model. 


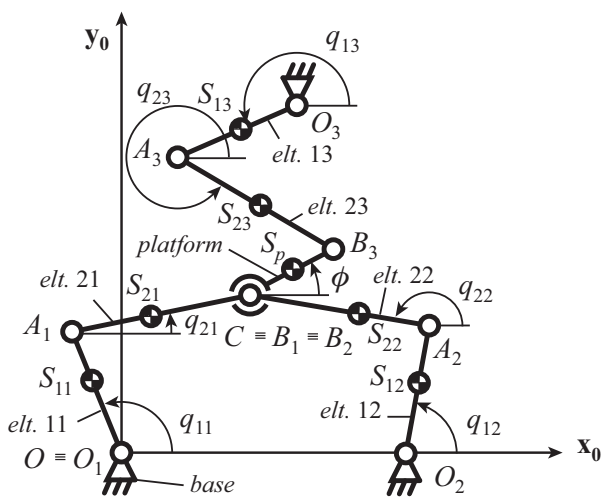

(a) Schematics

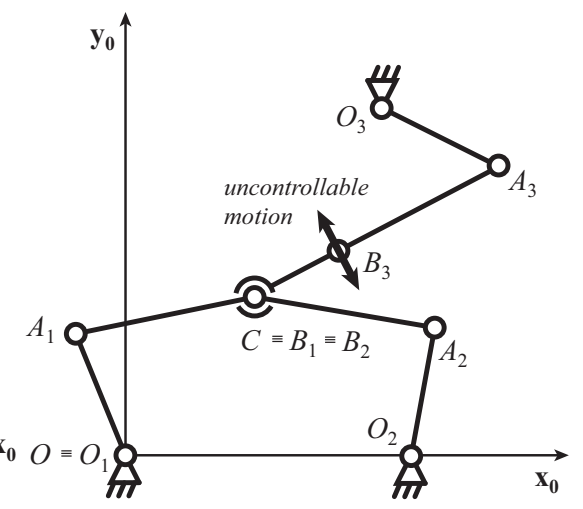

(b) Example of singularity

Figure 1: Schematics of the 3-RRR decoupled parallel robot and its singular configurations encountered during a full platform rotation.

\subsection{Dynamics of the robot}

In this part, the robot inverse dynamic model is computed using the Lagrange equations. To simplify the computation, it is preferable to replace the masses of moving links $2 i$ by concentrated masses (Seyferth, 1974). For a link $j i$ with mass $m_{j i}$ and its axial moment of inertia $I_{j i}$, we have:

$$
\left[\begin{array}{ccc}
1 & 1 & 1 \\
r_{j i} & 0 & r_{j i}-1 \\
r_{j i}^{2} L_{j i}^{2} & 0 & \left(r_{j i}-1\right)^{2} L_{j i}^{2}
\end{array}\right]\left[\begin{array}{l}
m_{j i 1} \\
m_{j i 2} \\
m_{j i 3}
\end{array}\right]=\left[\begin{array}{c}
m_{j i} \\
0 \\
I_{j i}
\end{array}\right]
$$

where:

- $r_{j i}$ is the non-dimensional position of the centre of masses $S_{j i}$ of element $j i$, i.e. for example for link $11, O S_{11}=r_{11} L_{11}$,

- $m_{j i k}(k=1,2,3)$ are the values of the three point masses placed at the centers of the revolute joints and at the center of masses of the link $j i$.

The robot kinetic energy can be expressed as:

$$
\begin{aligned}
2 T= & \sum_{i, j}\left(m_{j i} \mathbf{v}_{S_{j} i}^{2}+I_{j i} \dot{q}_{j i}^{2}\right)+m_{p}\left(\dot{x}^{2}+\dot{y}^{2}\right)+I_{p} \dot{\phi}^{2} \\
= & \sum_{i=1}^{3}\left(I_{r 1 i} \dot{q}_{1 i}^{2}+2 I_{r 2 i} \dot{q}_{1 i}\left(-\dot{x} \sin q_{1 i}+\dot{y} \cos q_{1 i}\right)\right)+m_{r p}\left(\dot{x}^{2}+\dot{y}^{2}\right) \\
& +I_{r p} \dot{\phi}^{2}+2 I_{r 3} \dot{q}_{13} \dot{\phi} \cos \left(q_{13}-\phi\right)+2 I_{r 4} \dot{\phi}(-\dot{x} \sin \phi+\dot{y} \cos \phi)
\end{aligned}
$$


where

$I_{r 1 i}=I_{1 i}+\left(m_{1 i} r_{1 i}^{2}+m_{2 i 1}+m_{2 i 2}\left(1-r_{2 i}\right)^{2}\right) L_{1 i}^{2}$,

$\left.I_{r 2 i}=m_{2 i 2} r_{2 i}\left(1-r_{2 i}\right)\right) L_{1 i}^{2}$,

$m_{r p}=m_{p}+m_{213}+m_{223}+m_{233}+m_{212} r_{21}^{2}+m_{222} r_{22}^{2}+m_{232} r_{23}^{2}$,

$I_{r p}=I_{p}+\left(m_{233}+m_{232} r_{23}^{2}\right) R^{2}$

$I_{r 3}=m_{232} r_{23}\left(1-r_{23}\right) R L_{13}$,

$I_{r 4}=\left(m_{p} r_{p}+m_{233}+m_{232} r_{23}^{2}\right) R$,

and $\dot{x}, \dot{y}$ are the platform translational velocities along $\mathbf{x}_{\mathbf{0}}$ and $\mathbf{y}_{\mathbf{0}}$ axes and

$\dot{\phi}$ is the platform rotation velocity, $\dot{q}_{1 i}$ is the joint rate of actuator $i, \mathbf{v}_{S_{j i}}$ is the velocity of the centre of masses $S_{j i}, \dot{q}_{j i}$ the rotational velocity of link $j i$, $m_{p}$ is the platform mass, $I_{p}$ its axial moment of inertia expressed at $C$ and $r_{p}$ the non-dimensional position of the centre of masses $S_{p}$ of the platform, i.e. $C S_{p}=r_{p} R$.

The gravity field is directed along the $\mathbf{z}_{\mathbf{0}}$ axis. As a result, the dynamic model can be computed as (Moon, 2007):

$$
\tau=\mathbf{w}_{\mathbf{b}}-\mathbf{B}^{T} \lambda, \text { with } \mathbf{A}^{T} \lambda=\mathbf{w}_{\mathbf{p}}
$$

where $\lambda$ are the Lagrange multipliers, matrices $\mathbf{A}$ and $\mathbf{B}$ are defined such that $\mathbf{A t}+\mathbf{B} \dot{\mathbf{q}}=\mathbf{0}$ for $\mathbf{t}=[\dot{x}, \dot{y}, \dot{\phi}]^{T}$ and $\dot{\mathbf{q}}=\left[\dot{q}_{11}, \dot{q}_{12}, \dot{q}_{13}\right]^{T}$ and

$$
\mathbf{w}_{\mathbf{b}}=\frac{d}{d t}\left(\frac{\partial T}{\partial \dot{\mathbf{q}}}\right)-\frac{\partial T}{\partial \mathbf{q}}, \mathbf{w}_{\mathbf{p}}=\frac{d}{d t}\left(\frac{\partial T}{\partial \mathbf{t}}\right)-\frac{\partial T}{\partial \mathbf{x}}, \mathbf{x}=[x, y, \phi]^{T}
$$

\section{Optimal Motion Generation}

\subsection{Condition for crossing the singularities}

As disclosed in (Briot and Arakelian, 2008), the singularities considered in this work can be crossed if and only if:

$$
\mathbf{t}_{s}^{T} \mathbf{w}_{\mathbf{p}}=0
$$

where $\mathbf{t}_{s}$ is the unit screw describing the gained degree of freedom when the robot encounters a singularity. For the robot at hand, for the 'orientation' singularity (Fig. 1b), it can be proven that $\mathbf{t}_{s}$ is defined by $\mathbf{t}_{s}=[0,0,1]^{T}$ throughout the workspace. Therefore, the condition for crossing this type of singularity remains to cancel the last line of $\mathbf{w}_{\mathbf{p}}$, i.e. from (2) and (4)

$$
I_{r p} \ddot{\phi}+I_{r 3}\left(\ddot{q}_{13} \cos \left(q_{13}-\phi\right)-\dot{q}_{13}^{2} \sin \left(q_{13}-\phi\right)\right)+I_{r 3}(\ddot{y} \cos \phi-\ddot{x} \sin \phi)=0,
$$

To lower the computation burden, it is preferable to express equation (6) as a function of $\dot{x}, \dot{y}, \dot{\phi}, \ddot{x}, \ddot{y}$ and $\ddot{\phi}$ only. The terms $\dot{q}_{13}$ and $\ddot{q}_{13}$ can be 
removed using the following kinematic relationships:

$$
\begin{aligned}
& \dot{\mathbf{q}}=-\mathbf{B}^{-1} \mathbf{A} \mathbf{t} \\
& \ddot{\mathbf{q}}=-\mathbf{B}^{-1}(\mathbf{A} \dot{\mathbf{t}}+\dot{\mathbf{A}} \mathbf{t}+\dot{\mathbf{B}} \dot{\mathbf{q}})=-\mathbf{B}^{-1}\left(\mathbf{A} \dot{\mathbf{t}}+\left(\dot{\mathbf{A}}-\dot{\mathbf{B}} \mathbf{B}^{-1} \mathbf{A}\right) \mathbf{t}\right)
\end{aligned}
$$

The values of $\dot{q}_{13}$ and $\ddot{q}_{13}$ can be extracted from the last line of vectors $\dot{\mathbf{q}}$ and $\ddot{\mathbf{q}}$.

\subsection{Illustrative example}

In order to validate the previous approach, an ADAMS model is developed with the following robot parameters extracted from (Chablat et al., 2012): $R=0.1 \mathrm{~m} ; L_{1 i}=L_{2 i}=0.33 \mathrm{~m} ; x_{O_{2}}=0.1 \mathrm{~m} ; x_{O_{3}}=0.05 \mathrm{~m}$; $y_{O_{3}}=0.6 \mathrm{~m} ; m_{1 i}=m_{2 i}=1 \mathrm{~kg} ; m_{p}=0.5 \mathrm{~kg} ; I_{1 i}=I_{2 i}=0.009 \mathrm{~kg} . \mathrm{m}^{2}$; $I_{p}=0.0017 \mathrm{~kg} \cdot \mathrm{m}^{2} ; r_{j i}=r_{p}=0.5$.

The robot is located at $\mathbf{x}\left(t_{0}\right)=\mathbf{x}_{\mathbf{i n}}=[-0.1 \mathrm{~m}, 0.45 \mathrm{~m}, 90 \mathrm{deg}]^{T}$ for $t_{0}=0 \mathrm{~s}$. Its task is to maintain the position of point $C$ while achieving a full rotation of the platform in $t_{f}=1.5 \mathrm{~s}$. Singularities will appear for $\phi_{s 1} \approx 269 \mathrm{deg}$ and $\phi_{s 2} \approx 328 \mathrm{deg}$. Two cases will be treated: (i) Case 1: a motion without taking into account the conditions for passing through the singularities, and (ii) Case 2: a motion that considers the conditions for passing through the singularities.

For Case 1, the motion planning is achieved with a 7 th degree polynomial law in order to cancel the velocity, acceleration and jerk at the beginning and at the end of the trajectory:

$$
\phi(t)=360 s(t)+90, s(t)=35\left(t / t_{f}\right)^{4}-84\left(t / t_{f}\right)^{5}+70\left(t / t_{f}\right)^{6}-20\left(t / t_{f}\right)^{7}
$$

where the following initial and final conditions are respected:

$s\left(t_{0}\right)=\dot{s}\left(t_{0}\right)=\dot{s}\left(t_{f}\right)=\ddot{s}\left(t_{0}\right)=\ddot{s}\left(t_{f}\right)=\dddot{s}\left(t_{0}\right)=\dddot{s}\left(t_{f}\right)=0, s\left(t_{f}\right)=1$.

The input torque of actuator 3 is plotted in Fig. 2a (it can be proven that if the platform position is not changed, the torques of actuators 1 and 2 are cancelled). It is apparent that the input torque tends to infinity at each singularity.

For Case 2, the motion is planned using three different polynomials $s_{k}$. Each part of the trajectory has to be achieved in $0.5 \mathrm{~s} . s_{1}$ is defined for $\phi \in\left[90 \mathrm{deg}, \phi_{s 1}\right]$ on the time interval $\left[t_{0}, t_{f 1}=0.5 \mathrm{~s}\right], s_{2}$ for $\phi \in\left[\phi_{s 1}, \phi_{s 2}\right]$ on the time interval $\left[t_{f 1}, t_{f 2}=1 \mathrm{~s}\right]$, and $s_{3}$ for $\phi \in\left[\phi_{s 2}, 450 \mathrm{deg}\right]$ on the time interval $\left[t_{f 2}, t_{f 3}=1.5 \mathrm{~s}\right]$ :

$$
\begin{aligned}
& \phi(t)=\left(\phi_{s 1}-90\right) s_{1}(t)+90, \text { for } t \in\left[t_{0}, t_{f 1}\right] \\
& \phi(t)=\left(\phi_{s 2}-\phi_{s 1}\right) s_{2}(t)+\phi_{s 1}, \text { for } t \in\left[t_{f 1}, t_{f 2}\right] \\
& \phi(t)=\left(450-\phi_{s 2}\right) s_{3}(t)+\phi_{s 2}, \text { for } t \in\left[t_{f 2}, t_{f 3}\right]
\end{aligned}
$$


with

$$
\begin{aligned}
s_{1}(t)= & 441.8819 t^{4}-2074.5386 t^{5}+3411.5717 t^{6}-1932.0441 t^{7}, \\
s_{2}(t)= & 0.1690 t+0.0159\left(t-t_{f 1}\right)^{2}+443.8291\left(t-t_{f 1}\right)^{4}-2116.1982\left(t-t_{f 1}\right)^{5} \\
& +3521.1306\left(t-t_{f 1}\right)^{6}-2011.4236\left(t-t_{f 1}\right)^{7}, \\
s_{3}(t)= & 2.4400\left(t-t_{f 2}\right)+3.8429\left(t-t_{f 2}\right)^{2}+15.8838\left(t-t_{f 2}\right)^{4} \\
& -316.3353\left(t-t_{f 2}\right)^{5}+746.8231\left(t-t_{f 2}\right)^{6}-506.5084\left(t-t_{f 2}\right)^{7},
\end{aligned}
$$

where the following initial and final conditions are respected in order to ensure the velocity, acceleration and jerk continuity all along the trajectory:

$$
\begin{aligned}
s_{1}\left(t_{0}\right) & =\dot{s}_{1}\left(t_{0}\right)=\ddot{s}_{1}\left(t_{0}\right)=\dddot{s}_{1}\left(t_{0}\right)=\dddot{s}_{1}\left(t_{f 1}\right)=0, s_{1}\left(t_{f 1}\right)=\dot{s}_{1}\left(t_{f 1}\right)=1, \\
\ddot{s}_{1}\left(t_{f 1}\right) & =0.0318 \\
s_{2}\left(t_{f 1}\right) & =\dddot{s}_{2}\left(t_{f 1}\right)=\dddot{s}_{2}\left(t_{f 2}\right)=0, s_{2}\left(t_{f 2}\right)=\dot{s}_{2}\left(t_{f 2}\right)=1, \ddot{s}_{2}\left(t_{f 2}\right)=7.6858 \\
\dot{s}_{2}\left(t_{f 1}\right) & =\dot{s}_{1}\left(t_{f 1}\right) d_{1}, \ddot{s}_{2}\left(t_{f 1}\right)=\ddot{s}_{1}\left(t_{f 1}\right) d_{1}, d_{1}=\left(\phi_{s 1}-90\right) /\left(\phi_{s 2}-\phi_{s 1}\right) \\
s_{3}\left(t_{f 2}\right) & =\dot{s}_{3}\left(t_{f 3}\right)=\ddot{s}_{3}\left(t_{f 3}\right)=\dddot{s}_{3}\left(t_{f 2}\right)=\dddot{s}_{3}\left(t_{f 3}\right)=0, s_{3}\left(t_{f 3}\right)=1 \\
\dot{s}_{3}\left(t_{f 2}\right) & =\dot{s}_{2}\left(t_{f 2}\right) d_{2}, \ddot{s}_{3}\left(t_{f 2}\right)=\ddot{s}_{2}\left(t_{f 2}\right) d_{2}, d_{2}=\left(\phi_{s 2}-\phi_{s 1}\right) /\left(450-\phi_{s 2}\right) .
\end{aligned}
$$

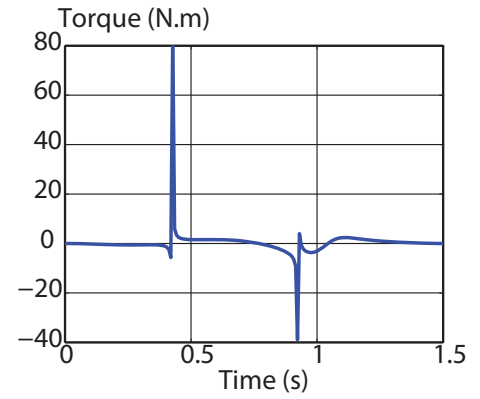

(a) For the trajectory defined at (8)

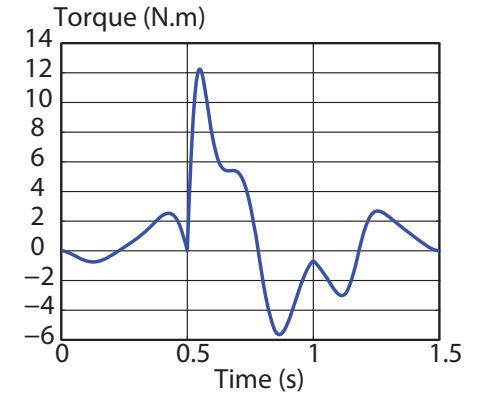

(b) For the trajectory defined at (9)

Figure 2: Torque of actuator 3.

It should be mentioned that the value of $\ddot{s}_{1}\left(t_{f 1}\right)\left(\ddot{s}_{2}\left(t_{f 2}\right)\right.$, resp.) is calculated so that it respects the condition for crossing the singularities expressed at $(6)$, taking into account that $\dot{s}_{1}\left(t_{f 1}\right)=1\left(\dot{s}_{2}\left(t_{f 2}\right)=1\right.$, resp. $)$. The value of $\phi$ all along this trajectory is plotted at Fig. 3a. Figure 3b presents the resulting displacement of actuator 3. It can be observed that this actuator has an oscillatory behaviour while the platform is ensuring a full rotation. 


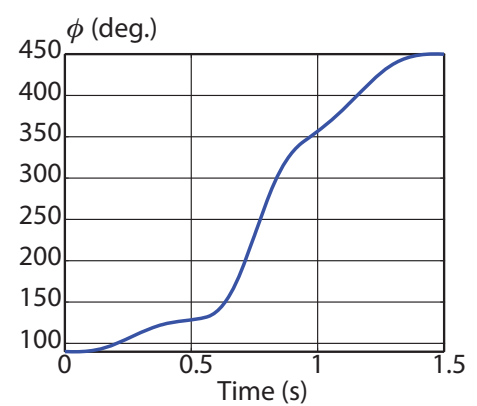

(a) Value of the platform rotation $\phi$

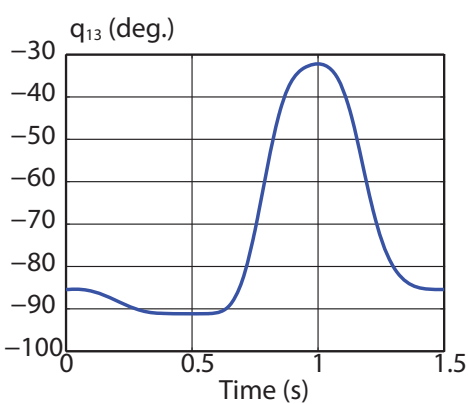

(b) Resulting displacement of actuator 3

Figure 3: The trajectory defined at (9).

The input torque of actuator 3 is plotted in Fig. 2b. It appears now that when crossing the singularities, the input torques remain finite.

It should be noted that in this example, only one turn has been achieved with a fixed position of point $C$, but it is obvious that the number of platform turns may be increased and that point $C$ can move as soon as the global trajectory respects equation (6) at each singular configuration.

\section{Conclusions}

It is known that the singularity leads to a loss of robot controllability and it is a serious obstacle for reproduction of stable motions. This drawback can be overcome by means of the optimum force control. In this paper, the optimal force generation of 3-RRR decoupled robots for ensuring unlimited platform rotation has been studied. The particularity of these robots is that an optimal choice of the legs' geometric parameters allows an unlimited platform rotation. However, a singular configuration is necessarily encountered during a full platform rotation. In order to generate a stable motion in the presence of singularities, optimal dynamic conditions were found. To illustrate the fruitfulness of the obtained results two different types of control have been compared: a motion generated via a 7 th degree polynomial law without taking into account the conditions for passing through the singularity and a motion that considers the optimal dynamic conditions for passing through the singularity. For the second case, a full rotation of the platform without perturbation of motion has been achieved. The obtained results showed that the generation of stable unlimited platform rotation of 3 -RRR decoupled robots is possible. It can be attractive for many industrial 
applications.

\section{Bibliography}

V. Arakelian, S. Briot, S. Yatsun, and A. Yatsun. A new 3-dof planar parallel manipulator with unlimited rotation capability. In Proc. 13th World Congress in Robot and Machine Science, 2011.

S. Briot and V. Arakelian. Optimal force generation of parallel manipulators for passing through the singular positions. International Journal of Robotics Research, 27:967-983, 2008.

D. Chablat, G. Moroz, V. Arakelian, S. Briot, and P. Wenger. Solution regions in the parameter space of a 3 -RRR decoupled robot for a prescribed workspace. In Proc. Advances in Robot Kinematics, submitted to, 2012.

O. Company, F. Pierrot, V. Nabat, and M. Rodriguez. Schoenflies motion generator: A new non redundant parallel manipulator with unlimited rotation capability. In Proceedings ICRA 2005, pages 3250-3255, 2005.

G. Gogu. Parallel mechanisms with decoupled rotation of the moving platform in planar motion. Journal of Mechanical Engineering Sciences, Part C, 224:709-720, 2009.

K.T. Kokikabushiki, F. Pierrot, and O. Company. 4-dof parallel robot, patent, 2000.

S. Lessard, P. Bigras, and I.A. Bonev. A new medical parallel robot and its static balancing optimization. Journal of Medical Devices, 1:272-278, 2007.

S. Lessard, I.A. Bonev, and Patent P. Bigras. Parallel manipulator, 2008.

X.-J. Liu, J. Wang, and G. Pritschow. A new family of spatial 3-dof fullyparallel manipulators with high rotational capability. Mechanism and Machine Theory, 40:475-494, 2005.

F.C Moon. Applied dynamics. J. Wiley and Sons, 2007.

V. Nabat, F. Pierrot, M.R. Mijangos, J.M.A. Arteche, R.B. Zabalo, O. Company, and K.F. Perez De Armentia. High-speed parallel robot with four degrees of freedom, patent, 2006a.

V. Nabat, F. Pierrot, M. Rodriguez, J.M. Azcoita, R. Bueno, O. Company, and K. Florentino. Unlimited-rotation parallel robot with four degrees of freedom, patent, 2006b.

S.E. Sacludean, L.J. Stocco, and I.C.N. Chau. 3-dof parallel manipulator, patent, 2002.

W. Seyferth. Massenersatz duch punktmassen in rumlichen getrieben. Mechanism and Machine Theory, 9:49-59, 1974. 